\title{
Interactive comment on "Intercomparison of total column ozone data from the Pandora spectrophotometer with Dobson, Brewer, and OMI measurements over Seoul, Korea” by Jiyoung Kim et al.
}

\section{Anonymous Referee \#2}

Received and published: 9 August 2016

\section{General comments}

The manuscript presents the results of a two-year comparison of total ozone measurements from Pandora, Brewer and Dobson spectrophotometers and satellite estimates (OMI, two algorithms). Results are given in terms of a least-squares analysis and some statistical indicators are provided ( $\max , \mathrm{min}$, average, standard deviation, relative differences, Pearson's correlation index, $R^{2}$ ) together with the analysis of variance (ANOVA). The manuscript addresses relevant questions and could potentially provide interesting results, since a complete set of instrument operates at the measuring station of Seoul. 
However, the manuscript is also disappointingly lacking of an appropriate analysis and looks just as a list of statistical indicators. Notably, a "Discussion" section examining in detail the sources of discrepancies is missing.

Indeed, it is stated that the differences between instruments (e.g., Pandora and Dobson) can be explained by a number of factors (e.g., SZAs, SO2, temperature, etc.), but no proof is provided. In a scientific paper, the authors should justify their statements based on a convincing analysis of a statistically representative number of data (e.g., SO2 measurements, effective ozone/instrumental temperatures, etc.). At least, the authors should provide a convincing proof that correcting for the above-mentioned factors of influence can improve the comparison and reduce the observed bias.

Also, the authors were not able to effectively disentangle the discrepancies arising from different sampling of datasets (on a daily or monthly basis) and those due to instrumental or processing issues.

Most of all, "very good agreement" among instruments is claimed, however differences are larger (up to $12.5 \%$, cf. line 367 ) than the stated uncertainties (e.g., compare Figs. 5-6 and Sects. 1-2), therefore this issue should be commented in detail.

The comparison between ground-based instruments and the OMI spaceborne radiometer is also too vague. How much do temporal and spatial resolutions affect the comparison? How much aerosols and other pollutants impact on satellite estimates of total ozone over Seoul?

In conclusion, the manuscript should not be published, in my opinion, unless major revisions are introduced.

\section{Specific comments}

No mention is made in the manuscript of the processing/algorithms of the measurements. Do they differ for different instruments? For example, how much can the used spectroscopic sets of cross-sections (and their relative dependence on effective ozone 
temperature) impact on the comparison?

Table 2: do the authors have an idea why some instruments have a negative intercept AMTD compared to Pandora and others a positive intercept?

How do the authors cope with straylight, which affects all data from the presented ground-based instruments? Lines 179-180 explain that measurements from Pandora at high SZAs are removed from the analysis, but what about the other instruments?

The comparison is performed in terms of monthly and daily averages. Then, a section (3.3) explains that large inconsistencies may originate due to the ozone variability when daily averages are performed on different datasets (because of different sampling frequencies). Could the authors additionally perform a comparison of nearlysimultaneous measurements? It is stated that the Pandora temporal resolution is about 2 minutes, therefore those measurements could be interpolated to the nearest Brewer/Dobson/OMI estimate, thus avoiding the bias illustrated in Sect. 3.3;

line 196: if the only condition for the comparison is that the number of daily observations must be greater than 10 days, it is likely that some differences are due to the sampled subset. Since the results orient the following comparison of the instruments, I think that the authors should revise their criteria or state the uncertainty of their results due to the day-to-day variability and different days sampled for each month in the datasets (as done in Sect. 3.3 for the daily averages);

The dependence on solar zenith angle is listed as an important factor impacting the comparison (line 365). Could the authors present some plots of the differences between instruments as a function of the solar zenith angle or the airmass?

What is the expected magnitude of the SO2 effect in Seoul (line 365)?

Printer-friendly version

\section{Technical corrections}

- line 5 (and 209): it should be clarified why Pandora is taken as a reference for the comparison. Is it because of its high temporal resolution? In this case, this should be

Interactive

comment

\section{Discussion paper}


explicitly stated;

- line 10: reporting the slope and $\mathrm{R}^{2}$ for the comparison with the Brewer is redundant, since these data are already provided few lines above;

- line 12-13: are both instruments affected by these factors in a similar way?

Interactive

- line 13: does "temperature" mean effective ozone temperature or instrumental temcomment perature? Or both?

- Sect. 1: I would suggest to merge the description of the instruments in Sect. 1 with the text in Sect. 2. Instead, some references about previous comparisons of ozone instruments should be added in Sect. 1;

- line 25-27: please, add some bibliographic references;

- line 31: the recovery of the "ozone hole" is still an open question (e.g., Solomon et al., Science, 2016), and the cited articles (1997-2003) do not pretend to report the recovery of the ozone hole, contrary to what the authors state. Please, notice that slowdown of depletion does not necessarily mean recovery. Moreover, a quite confusing explanation of the "ozone hole" is offered to the reader (without even specifying where it occurs) and no mention to the decrease of ozone at midlatitudes is made. Please, rewrite this part;

- line 53: $363 \mathrm{~nm}$. Is the Brewer an "extended" Brewer (MkIV-e)? If not, the readers might wonder why a single Brewer is able to measure at such large wavelengths;

- line 69: is the word "error" used in place of "uncertainty"?

- line 94: why "An" OMI?

- lines 121-127: I don't understand what this short review of previous investigations have to do with the aim of the manuscript. Please, focus on Brewer functioning and processing, instead;

Printer-friendly version

Discussion paper 
- line 216: how can the authors state that Fig. 3 shows a "generally gaussian distribution" based on only 6 bins? Can they support their sentence on the basis of a normality test?

- line 221: "Dobson unit" or "Dobson instrument"?

- line 224: are you comparing Pandora, Brewer and OMI from 2012-2014 to the Dobson in the period 1985-2000? Please rewrite this sentence, since it is very confusing. Furthermore, can you assess an increase/decrease by comparing datasets from different instruments?

- lines 261-266: please, provide a short explanation about the additional information that can be extracted from the ANOVA analysis and the meaning of the ANOVA outputs that are presented in the text;

- lines 306-313: since the error sources are a key point of the comparison, this part should be commented in detail rather than just providing bibliographic citations;

- lines 306-309: what is the reason of the observed SZA-dependence?

- lines 312: "fixed temperature". Do you mean effective ozone temperature? Does humidity refer to instrumental humidity or atmospheric humidity?

- line 365: "temperature". Instrumental or ozone effective?

- Table 1: please, write more clearly the year (2012-2013) and the day;

- Fig. 4: define the used acronyms (OMT, OMD, DBS, etc.). Also, Fig. 4d has no dashed lines. Explain in the caption what the dashed lines indicate;

- Fig. 5, caption: "blue lines". There are no blue lines in the figures;

- Figs. 4-5: why $n=115$ for all plots? Only days with all instruments measuring were chosen? Is it necessary to report the same number in all plots?

Printer-friendly version

Interactive comment on Atmos. Meas. Tech. Discuss., doi:10.5194/amt-2016-146, 2016.

Interactive

comment

C5

Discussion paper 